\section{GENRE}

en séries

\section{Genre en séries}

Cinéma, télévision, médias

5 | 2017

Masculinités imag(in)les 2

\title{
La masculinité comme spectacle. Réflexions sur les hommes et le cinéma dominant
}

Traduction de Neale, Steve (1993 [2005]) « Masculinity as spectacle : Reflections on Men and Mainstream Cinema », dans Cohan \& Hark (eds.) Screening The Male : Exploring Masculinity in Hollywood Cinema

\section{Steve Neale}

Traducteur : Charles-Antoine Courcoux et Geneviève Sellier

\section{OpenEdition}

Journals

Édition électronique

URL : http://journals.openedition.org/ges/896

DOI : $10.4000 /$ ges.896

ISSN : 2431-6563

Éditeur

Presses universitaires de Bordeaux

Référence électronique

Steve Neale, "La masculinité comme spectacle. Réflexions sur les hommes et le cinéma dominant », Genre en séries [En ligne], 5 | 2017, mis en ligne le 01 juin 2017, consulté le 22 mars 2021. URL : http:// journals.openedition.org/ges/896 ; DOI : https://doi.org/10.4000/ges.896

Ce document a été généré automatiquement le 22 mars 2021.

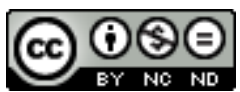

La revue Genre en séries est mise à disposition selon les termes de la Licence Creative Commons Attribution - Pas d'Utilisation Commerciale - Pas de Modification 4.0 International. 


\section{La masculinité comme spectacle. Réflexions sur les hommes et le cinéma dominant}

Traduction de Neale, Steve (1993 [2005]) « Masculinity as spectacle : Reflections on Men and Mainstream Cinema », dans Cohan \& Hark (eds.) Screening The Male : Exploring Masculinity in Hollywood Cinema

\section{Steve Neale}

Traduction : Charles-Antoine Courcoux et Geneviève Sellier

1 Dans les dix dernières années, de nombreux livres et d'articles sont parus sur les images de femmes produites et diffusées par l'institution cinématographique. Motivés politiquement par le développement du Mouvement des Femmes, et attentifs par conséquent aux implications politiques et idéologiques des représentations des femmes au cinéma, plusieurs de ces ouvrages et articles se sont appuyés sur le texte de Laura Mulvey "Plaisir visuel et cinéma narratif ", publié par Screen en 1975. L'article de Mulvey a eu beaucoup d'influence dans la manière dont il a articulé une perspective psychanalytique sur le cinéma avec une perspective féministe axée sur la façon dont les images de femmes sont configurées dans les films tout public. Elle a cherché à montrer à quel point les mécanismes psychiques que le cinéma met en jeu sont profondément patriarcaux, et à quel point les images de femmes que le cinéma dominant a produites sont au cœur de ces mécanismes.

Dès lors, chaque fois qu'il y a eu des discussions sur le genre (gender), la sexualité, les représentations et le cinéma au cours de la dernière décennie, cette discussion a eu tendance à être centrée sur la représentation des femmes, et à tirer ses principes fondamentaux de l'article de Mulvey. C'est seulement au sein du mouvement gay que sont apparues des discussions spécifiques sur la représentation des hommes. La plupart, pour autant que je sache, ont été centrées sur les représentations et les stéréotypes concernant les homosexuels. Tant dans le mouvement féministe que dans le mouvement gay, on a l'impression persistante que les images et les fonctions de la masculinité hétérosexuelle dans le cinéma dominant ont été laissées en dehors des 
débats. La masculinité hétérosexuelle a été identifiée comme une norme structurelle par rapport aux images des femmes et des gays. À ce titre, elle a été profondément problématisée et rendue visible. Mais elle a rarement été discutée et analysée en tant que telle. Et encore moins en dehors de ces mouvements. Il est ainsi très rare de trouver des analyses qui visent à spécifier en détail, en relation avec un film particulier ou un groupe de films, comment la masculinité hétérosexuelle est configurée, et les mécanismes, tensions et contradictions que cette configuration peut impliquer. Mis à part un certain nombre de textes récents dans Screen (Cook, 1982 ; Neale, 1982 ; Caughie et Skirrow, 1982 ; Modleski, 1982) et Framework (Willemen, 1981), l'article de Raymond Bellour sur La Mort aux trousses (Bellour, 1975) est le seul exemple qui vient immédiatement à l'esprit. L'article de Bellour décrit en détail la trajectoire œdipienne du film d'Hitchcock, en retraçant le parcours de son protagoniste, Roger Thornhill (Cary Grant), depuis sa position de dépendance infantile par rapport à sa mère jusqu'à une posture masculine "adulte", "virile", hétérosexuelle, scellée par son mariage avec Eve Kendall (Eva Marie Saint) et par son acceptation du rôle et de l'autorité du père. Cependant, l'article s'intéresse autant au travail du film classique hollywoodien en général qu'aux spécificités d'un ensemble d'images de la masculinité.

Bien qu'il y ait par conséquent un réel besoin de plus d'analyses de films particuliers, j'entends dans cet article adopter une autre approche pour traiter ces questions. En utilisant l'article de Mulvey comme une référence centrale et structurante, je vais me pencher en particulier sur les notions d'identification, de regard et de spectacle qu'elle a traitées et m'interroger sur la façon dont ses remarques s'appliquent directement ou indirectement aux images d'hommes, d'une part, et au spectateur masculin d'autre part. L'objectif est moins de contester fondamentalement les thèses qu'elle avance que d'ouvrir un espace dans le cadre de son argumentation et de ses remarques pour prendre en considération les représentations de la masculinité, dans la mesure où cette dernière peut être mise en relation avec les caractéristiques et les conventions de l'institution cinématographique.

\section{Identification}

Pour commencer, je voudrais citer le livre de John Ellis, Fictions visibles (1982). Écrit à la lumière de l'article de Mulvey, le livre d'Ellis cherche à la fois à tirer parti de ses arguments et à étendre et qualifier certaines de ses thèses à propos du genre (gender) et de l'identification au cinéma. Ellis fait valoir que l'identification n'est jamais simplement une question d'identification des hommes avec des figures masculines à l'écran et des femmes qui s'identifieraient avec des figures féminines. Le cinéma exploite et implique plusieurs désirs et plusieurs formes de désir. Et le désir lui-même est mobile, fluide, transgressant constamment les identités, les positions et les rôles. Les identifications sont multiples, fluides et même, dans une certaine mesure, contradictoires. Qui plus est, il y a différentes formes d'identification. Ellis pointe deux formes en particulier, l'une associée au narcissisme, l'autre aux fantasmes et aux rêves. Il résume ainsi :

L'identification au cinéma comprend deux tendances différentes. D'abord, il y a celle du rêve et des fantasmes qui implique des tendances multiples et contradictoires dans la construction de l'individu. Ensuite, il y a l'expérience de l'identification narcissique avec l'image d'une figure humaine perçue comme autre. Ces deux processus sont mobilisés par les conditions du cinéma de divertissement. 
Le spectateur ne s'identifie donc pas avec le héros ou l'héroïne : une identification qui impliquerait, si on la prend dans le sens commun, que les personnes construites socialement comme des hommes s'identifient aux héros masculins, alors que celles construites socialement comme des femmes s'identifient aux héroïnes. La situation est plus complexe que cela, car l'identification implique à la fois la reconnaissance de soi dans l'image sur l'écran, une identification narcissique, et l'identification de soi avec les différentes positions qui sont impliquées dans la narration fictionnelle : celles du héros et de l'héroïne, le méchant, le figurant, le personnage actif et le personnage passif. L'identification est donc multiple et fracturée, comme si le spectateur/ la spectatrice regardait les différentes composantes de sa psyché parader devant elle ou lui. (Ellis, $1982: 43$ )

Une série d'identifications sont mises en jeu, pour être ensuite changeantes et mobiles. De même, cependant, un constant travail s'effectue pour canaliser et réguler l'identification en rapport avec la dichotomie sexuée, en rapport avec les ordres du genre, de la sexualité et de l'identité sociale, et avec l'autorité, qui caractérisent la société patriarcale. Chaque film tend à la fois à adopter et à travailler activement au renouvellement de cet ordre, de cette dichotomie. Chaque film tend donc à configurer l'identification en fonction des catégories socialement définies et construites de l'homme et de la femme.

6 En me focalisant spécifiquement sur la masculinité dans ce contexte, je vais tenter d'analyser plus en détail le processus de l'identification narcissique. Dans la mesure où les films impliquent une identification de genre, et où les idéologies actuelles de la masculinité engagent de façon si centrale des notions et des attitudes relatives à l'agression, au pouvoir et au contrôle, il me semble que le narcissisme et l'identification narcissique pourraient s'avérer particulièrement significatifs.

7 Le narcissisme et l'identification narcissique impliquent tous deux des fantasmes de pouvoir, d'omnipotence, de maîtrise et de contrôle. Laura Mulvey fait le lien entre ces fantasmes et les images patriarcales de la masculinité dans les termes suivants :

Lorsque le spectateur s'identifie au protagoniste masculin principal, il projette son regard sur celui de son semblable, son substitut à l'écran, de sorte que le pouvoir du héros, qui contrôle les évènements, coïncide avec le pouvoir actif du regard érotique, les deux donnant un sentiment satisfaisant d'omnipotence. Ainsi, une star masculine de cinéma n'est pas séduisante parce qu'elle est l'objet érotisé de son regard, mais parce qu'elle possède les mêmes caractéristiques que le moi idéalisé, plus parfait, plus complet et plus puissant, construit lors du stade du miroir ${ }^{1}$. (Mulvey, 1975)

8 Je vais revenir sur les remarques de Mulvey à propos du glamour des stars masculines plus loin. Mais d'abord il est utile d'étendre et d'illustrer son idée sur le protagoniste masculin et jusqu'à quel point son image dépend de fantasmes narcissiques d'un « idéal du moi plus parfait, plus complet, plus puissant. »

9 Il est assez facile de trouver des exemples de films dans lesquels ces fantasmes sont largement répandus, où le héros est puissant et omnipotent à un degré extraordinaire : le personnage de Clint Eastwood dans Une poignée de dollars, Et pour quelques dollars de plus, et dans Le Bon, la brute et le truand, les westerns de Tom Mix, Charlton Heston dans Le Cid, la série des Mad Max, les films épiques de Steve Reeves, Superman, Flash Gordon, et ainsi de suite. En général, évidemment, ce sont des drames où le pouvoir et l'omnipotence sont mis à l'épreuve et confirmés (Superman 2 est un exemple particulièrement intéressant, tout comme les westerns et les films d'aventure de Howard Hawks), mais la trilogie de Sergio Leone, par exemple, se caractérise par 
l'étendue du pouvoir du héros qui va jusqu'à ressembler à un pouvoir divin, difficilement qualifiable. De là, peut-être, leur construction autour de scènes ritualisées qui, à de nombreux égards, sont dénuées de véritable suspense. D'un autre côté, un film comme Le Samouraï de Melville commence avec l'image d'une masculinité maîtresse d'elle-même, toute puissante, et retrace sa désintégration graduelle et finale. Alain Delon joue un gangster solitaire, un tueur à gages. Son propre narcissisme est souligné par son souci obsessionnel de son apparence, marqué notamment par le geste répétitif et ritualisé qu'il fait lorsqu'il met son chapeau, la main glissant sur le bord. Delon a un contrat à accomplir, mais il est repéré par une chanteuse noire dans un club. Il y a un échange de regards. À partir de ce moment-là, son omnipotence, son silence et son invulnérabilité sont tous menacés. On lui tire dessus et il est blessé ; son appartement est cambriolé et mis sens dessus dessous; il est presque piégé dans le métro. Finalement, il est tué, après être retourné au club pour revoir la chanteuse. Le film n'est aucunement une critique de l'image masculine qu'il construit. Au contraire, il s'identifie complètement (et nous invite à nous identifier) à Delon. Néanmoins, le film déploie clairement les éléments de cette image et ceux qui la rendent vulnérable. Ce n'est pas un hasard si la chute de Delon commence symptomatiquement par sa rencontre avec une femme noire. La différence (la double différence) est la menace. L'expression de la menace, c'est cet échange de regards où le regard d'autorité froide de Delon est troublé, miné et retourné.

Le genre d'image que Delon incarne ici, ou celle d'Eastwood et des autres acteurs mentionnés précédemment, se caractérisent non seulement pas une retenue émotionnelle mais aussi par le silence, une réticence au langage. Théoriquement, ce silence, cette absence de paroles peut encore être associée au narcissisme et à la construction d'un moi idéal. L'acquisition du langage est un processus profondément en lutte avec le narcissisme dans la petite enfance. Cela participe de ce qu'on appelle la «castration symbolique ». Le langage est un processus (ou un ensemble de processus) qui implique l'absence et le manque et c'est ce qui menace l'image de soi comme totalement fermée, autosuffisante, omnipotente. La construction d'un moi idéal, en attendant, est un processus impliquant de profondes contradictions. Alors que le moi idéal peut être un modèle auquel le sujet s'identifie et auquel il aspire, il peut être aussi une source d'images et de sentiments de castration, dans la mesure où cet idéal est quelque chose avec lequel le sujet n'est jamais en adéquation (Safouan, 1981 : 85-87).

11 Si c'est le cas, il ne peut pas y avoir d'identification simple et non problématique de la part du spectateur ou de la spectatrice, avec le «moi idéal» sur l'écran, dont parle Mulvey. Dans un article publié dans Wide Angle, David N. Rodowick fait un constat similaire. Il poursuit en arguant que l'image narcissique masculine - l'image d'autorité et d'omnipotence - peut entraîner une forme de masochisme concomitant dans les relations entre le spectateur et l'image, et en outre que l'image masculine peut comporter un certain érotisme, dans la mesure où il y a toujours une oscillation constante entre l'image comme source d'identification et en tant qu'autre, comme objet de contemplation. L'image est à la fois la source de processus et de conduites narcissiques et, dans la mesure où elle est un autre, la source de processus et de conduites orientés vers l'objet :

Mulvey discute de la star masculine en tant qu'objet du regard mais lui dénie toute fonction en tant qu'objet érotique. Parce que Mulvey conçoit le regard comme fondamentalement actif dans ses buts, l'identification avec le protagoniste masculin n'est prise en compte que d'un point de vue qui est associé à un sentiment 
d'omnipotence, qui endosse le contrôle du récit. Elle ne fait aucune différence entre l'identification et le choix d'objet qui peut être orienté avec une dimension sexuelle vers la figure masculine, ni ne prend en considération la signification de l'autorité dans la figure masculine du point de vue d'une économie masochiste. (Rodowick, $1982: 8)$

12 Compte tenu de l'argumentation de Rodowick, il n'est pas surprenant que les genres et les films «masculins" comportent constamment des thèmes, des scènes et des fantasmes sadomasochistes, ni que le héros masculin soit de temps en temps construit comme l'objet d'un regard érotique. Ce sont ces deux points que je souhaiterais discuter maintenant. Toutefois, il est important de mentionner ici qu'ils ont été déjà discutés par Paul Willemen dans son article intitulé « Anthony Mann : un regard sur l'homme » (Willemen, 1981).

13 Willemen soutient que le spectacle et le schéma dramatique dans les films de Mann tendent tous deux à être structurés autour du regard sur la figure masculine: "L'expérience du spectateur est basée sur le plaisir de voir 'exister' un homme (c'est-àdire marcher, bouger, monter à cheval, se battre) dans ou à travers les paysages urbains, sauvages, ou plus abstraitement, l'Histoire ; et sur le plaisir angoissant de voir un homme mutilé (de façon souvent très crue chez Mann) et restauré avec brutalité et violence.» (Willemen, 1981: 16). Ces plaisirs sont fondés sur un voyeurisme homosexuel réprimé, un voyeurisme «qui n'est pas sans problèmes: le fait de contempler un homme produit autant d'anxiété que le fait de regarder une femme, surtout quand c'est présenté de façon aussi directe que dans les scènes de meurtre dans La Brigade du suicide (T-Men) et Incident de frontière (Border Incident) (Willemen, 1981 : 16). La thèse (implicite) derrière ces commentaires semble être que, dans une société hétérosexuelle et patriarcale, le corps masculin ne peut pas être explicitement désigné comme l'objet érotique d'un autre regard masculin ; ce regard doit être motivé d'une autre façon, et son contenu érotique réprimé. Les mutilations et le sadisme si souvent présents dans les films de Mann sont des marques à la fois de la répression et des moyens par lesquels le corps masculin peut être disqualifié, pour ainsi dire, comme objet de contemplation et de désir érotiques. La répression et le désaveu que cela implique sont visibles dans les scènes de T-Men et de Border Incident auquel Willemen se réfère, où « un agent infiltré doit regarder, impassible, pendant que son meilleur ami et partenaire se fait tuer » (Willemen, $1981: 16)$.

Il y a une dernière contradiction importante dans ce type d'images narcissiques de masculinité, que je voudrais mettre en évidence. C'est la contradiction entre le narcissisme et la Loi, entre une image d'autorité narcissique d'un côté et une image d'autorité sociale de l'autre. Cette tension ou contradiction est longuement discutée par Laura Mulvey dans un article visant à considérer à nouveaux frais « Plaisir visuel » en analysant Duel au soleil (Mulvey, 1981). C'est une tension qu'elle considère comme particulièrement évidente dans le western. En utilisant un modèle dérivé de l'analyse des contes populaires par Vladimir Propp (1970), Mulvey souligne deux fonctions narratives, le «mariage » (et donc l'intégration sociale) et le "non-mariage », le refus du héros de s'intégrer socialement, refus motivé par un narcissisme nostalgique :

Dans le conte proppien, un aspect important de la clôture narrative est le "mariage », une fonction figurée par la "princesse » ou un équivalent. C'est la seule fonction qui est spécifique au sexe et donc associée au sexe du héros et à sa «mariabilité ». Cette fonction est très fréquente dans le western, où le mariage contribue de manière décisive à la clôture narrative. Cependant, la présence de cette fonction en est aussi venue à introduire un élément de complexité dans le 
western, à travers son opposé complémentaire, le «non-mariage ». En effet, alors que l'intégration sociale représentée par le mariage est un aspect essentiel du conte populaire, dans le western il peut avoir lieu... ou pas. Le héros peut gagner en stature en refusant la princesse et rester seul (Randolph Scott dans la série des films de Budd Boetticher). Étant donné que la fin du conte proppien peut être interprétée comme représentant la résolution du complexe d'Ædipe (l'intégration dans l'ordre symbolique), le rejet du mariage symbolise la célébration nostalgique de l'omnipotence phallique et narcissique. (Mulvey, 1981 : 14)

Il y a donc deux images divergentes de la masculinité couramment en jeu dans le western :

La tension entre deux points d'attraction, le symbolique (l'intégration sociale et le mariage) et le narcissisme nostalgique, provoque la division du héros de western entre les deux, ce qu'on ne trouve pas dans le conte proppien. Ici, deux fonctions émergent, l'une célébrant l'intégration dans la société grâce au mariage, l'autre célébrant la résistance aux normes et aux responsabilités sociales, et par-dessus tout au mariage et à la famille, sphère représentée par les femmes. (Mulvey, 1981 :

18)

Mulvey en vient à discuter du western de John Ford L'Homme qui tua Liberty Valance, et note l'opposition entre Tom Doniphon, joué par John Wayne, qui incarne la fonction narcissique du marginal socialement anachronique, et Ransom Stoddart, joué par James Stewart, qui incarne la fonction civilisatrice du mariage, de l'intégration et de la responsabilité sociales. Le ton du film est de plus en plus nostalgique, en phase avec le deuil de la perte de Tom Doniphon et de ce qu'il représente. La nostalgie n'est donc pas seulement celle d'un passé historique, de l'Ouest ancien, mais aussi celle du narcissisme masculin que représente Wayne.

En prenant en compte les remarques de Mulvey sur la nostalgie dans Liberty Valance, on pourrait discuter d'un grand nombre de westerns dans les mêmes termes, via le thème de la perte ou de la condamnation du narcissisme masculin. Les exemples les plus clairs seraient les westerns de Peckinpah: Coups de feu dans la sierra, Major Dundee (dans une moindre mesure), La Horde sauvage, et surtout Pat Garrett et Billy the Kid. Ces films sont imprégnés de nostalgie, avec une obsession pour les images et les définitions de la masculinité et des codes masculins de comportement, avec des images de narcissisme masculin face aux menaces que représentent les femmes, la société et la Loi. La menace de castration est figurée par les plaies et les blessures dont souffrent Joel McCrea dans Coups de feu dans la sierra, Charlton Heston dans Major Dundee et William Holden dans La Horde sauvage. Les fameux ralentis dans les séquences violentes, avec les corps qui volent en éclat et sont déchiquetés, peuvent être interprétés à un certain niveau comme des images narcissiques dans leur moment de désintégration et de destruction. Significativement, Kris Kristofferson dans le rôle de Billy dans Pat Garrett et Billy the Kid, l'ultime incarnation du narcissisme masculin omnipotent dans les films de Peckinpah, se voit épargner une mort sanglante et mutilante. Tué par Pat Garrett, son corps ne montre aucun signe de blessure ni de sang : le narcissisme est transfiguré (au lieu d'être détruit) par la mort.

Je vais à présent passer des questions d'identification et de narcissisme à une discussion en relation avec les images d'hommes et la masculinité sur les deux modes de regard identifiés par Mulvey dans « Plaisir visuel », le regard voyeuriste d'une part et le regard fétichiste de l'autre. 


\section{Le regard et le spectacle}

19 En discutant de ces deux types de regards, tous deux fondamentaux au cinéma, Mulvey les situe seulement en relation avec une structure d'activité/passivité dans laquelle le regard est masculin et actif alors que l'objet du regard est féminin et passif. Les deux sont considérés comme des moyens distincts et variés par lesquels les angoisses masculines de castration peuvent être mises en jeu et évacuées.

Le regard voyeuriste est repérable pour autant qu'il y ait une distance entre le spectateur et le spectacle, par un fossé entre le voyeur et le l'objet du regard.

Cette structure permet au spectateur d'avoir un certain pouvoir sur ce qu'il voit. Par conséquent, cela tend constamment à générer des fantasmes et des thématiques sadomasochistes. Voici la description de Mulvey :

Le voyeurisme... a des associations avec le sadisme: le plaisir réside dans

l'évaluation d'une culpabilité (immédiatement associée à la castration),

l'affirmation d'un contrôle et la soumission du coupable à la punition ou au pardon.

Ce côté sadique colle parfaitement au récit. Le sadisme exige une histoire, il faut

qu'il arrive quelque chose, qu'un changement soit provoqué chez quelqu'un, qu'il y

ait un affrontement entre la force et la volonté, une victoire et une défaite, tout cela

se produisant dans un temps linéaire avec un début et une fin. (Mulvey, 1975 : 14)

Mulvey discute ensuite des caractéristiques du regard voyeuriste dans les termes du film noir et des films d'Hitchcock, où le héros est le porteur du regard voyeuriste, engagé dans une histoire où la femme est la cible de ses composantes sadiques. Cependant, si l'on prend certains des termes utilisés dans sa description - «faire arriver quelque chose", "provoquer un changement chez quelqu'un", "un affrontement entre la volonté et la force ", " une victoire et une défaite »-, ils peuvent s'appliquer immédiatement aux genres "masculins", aux films concernés en grande partie ou uniquement par la description des relations entre hommes, à n'importe quel film, par exemple, où il $\mathrm{y}$ a un affrontement entre le héros masculin et le vilain. Les films de guerre, les westerns, les films de gangsters, par exemple, sont tous caractérisés par «l'action», par «faire que quelque chose arrive». Les batailles, les combats et les duels de toute sorte renvoient à des affrontements de "la volonté et de la force », des victoires et des défaites, entre des hommes individuels ou en groupe. Tout cela implique que les figures masculines à l'écran soient soumises à un regard voyeuriste, tant de la part des spectateurs que des autres personnages masculins.

La thèse de Paul Willemen sur les films d'Anthony Mann est très pertinente ici. La répression de tout aveu explicite d'érotisme dans l'acte de regarder un homme semble structurellement liée à un contenu narratif caractérisé par des fantasmes et des scènes sadomasochistes. Par conséquent, les deux formes de regard voyeuriste, intra et extradiégétique, sont particulièrement évidentes dans les moments d'affrontement ou de combat mentionnés ci-dessus, où l'issue narrative est déterminée par un corps à corps ou par une fusillade, quand l'affrontement entre hommes devient un pur spectacle. Peut-être les exemples les plus extrêmes sont-ils dans les westerns de Leone, où l'échange de regards agressifs, qui caractérise tant de duels d'armes à feu, est poussé jusqu'à la parodie via l'usage répétitif de très gros plans. À ce moment, le regard commence à osciller entre le voyeurisme et le fétichisme pendant que l'histoire est suspendue pour céder la place au spectacle. Les aspects angoissants du regard sur l'homme auquel Willemen se réfère, sont ici à la fois représentés et apaisés, pas 
seulement en déployant le sadisme inhérent au voyeurisme à travers les scènes de violence et de combat, mais aussi en utilisant les structures et le processus du regard fétichiste, en interrompant le récit pour reconnaître le plaisir de l'exhibition, mais en le déplaçant du corps masculin en tant que tel pour le localiser plus largement dans l'ensemble des composants d'une scène hautement ritualisée.

John Ellis a caractérisé le regard fétichiste dans les termes suivants :

Alors que le voyeurisme maintient (et dépend de) une séparation entre le voyeur et l'objet regardé, le fétichisme tente d'abolir ce fossé. [...] Ce processus implique une position et un comportement différents du spectateur par rapport à l'image. Il représente la tendance opposée au voyeurisme. [...] Le regard fétichiste implique la reconnaissance directe et la participation de l'objet regardé [...] Dans l'attitude fétichiste, le regard du personnage sur celui qui le regarde [...] est un trait central. [...] Le regard voyeuriste est curieux, inquisiteur, il cherche à savoir. Le regard fétichiste est captivé par ce qu'il voit et ne désire pas en savoir davantage, voir plus, découvrir. [...] Le regard fétichiste se contente de l'exhibition et du spectaculaire. (Ellis, 1982:47)

25 À nouveau, Mulvey discute de façon centrale de la forme de ce regard en relation avec la femme comme objet: "Ce second moyen, la scopophilie fétichiste, construit la beauté physique de l'objet, et en fait quelque chose de satisfaisant en soi. " (Mulvey, 1981 : 14). La "beauté physique » est interprétée exclusivement en termes de corps féminin. C'est précisé à travers l'exemple des films de Sternberg:

Alors qu'Hitchcock se penche sur la dimension investigatrice du voyeurisme, Sternberg produit le fétiche ultime, le hissant jusqu'au point où la puissance $d u$ regard du protagoniste masculin se brise au profit de l'image elle-même, dans un rapport érotique direct avec le spectateur. La beauté de la femme comme objet et l'espace de l'écran fusionnent; elle n'est plus porteuse de la culpabilité mais un produit parfait, dont le corps, stylisé et fragmenté par les gros plans, est le contenu même du film, et le destinataire direct du regard du spectateur. (Mulvey, $1981: 14$ )

Si nous revenons aux scènes de fusillade de Leone, nous constatons que certains éléments du regard fétichiste tels qu'ils sont décrits ici, sont présents, et d'autres pas. On nous offre le spectacle de corps masculins, mais des corps qui ne sont pas marqués en tant qu'objet d'exhibition érotique. Il n'y a aucune trace d'une reconnaissance ou de la conscience que ces corps sont exhibés uniquement pour le regard du spectateur. Ils sont exhibés, certes, mais il n'y a pas de convention culturelle ou cinématographique qui permette au corps masculin de se présenter dans les termes où Dietrich l'est si souvent dans les films de Sternberg. Nous voyons des corps masculins stylisés et fragmentés par des gros plans, mais notre regard n'est pas direct, il est fortement médiatisé par le regard des personnages impliqués dans la scène. Et ces regards ne sont pas caractérisés par le désir, mais plutôt par la peur, la haine ou l'agressivité. Les fusillades sont des moments de spectacle, des moments où le récit hésite, où il s'arrête momentanément, mais ce sont aussi des moments où le drame finit par se résoudre, un temps de suspense au point culminant de l'enchaînement narratif. Ces scènes impliquent ainsi une imbrication des deux formes de regard, leur entrelacement visant à minimiser et à déplacer l'érotisme qu'ils tendent tous deux à véhiculer, pour désavouer tout regard explicitement érotique sur le corps masculin.

Il y a d'autres types d'affrontement masculin qui semble fonctionner de cette façon. Mis à part le western, on pourrait mentionner le genre épique, du combat de gladiateurs dans Spartacus, jusqu'au combat entre Christopher Plummer et Stephen Boyd à la fin de La Chute de l'Empire romain, en passant par la course de chars dans Ben Hur. On peut 
trouver des représentations plus directes du corps masculin, bien qu'elles tendent à être soit plutôt brèves soit à occuper l'écran pendant le générique de fin ou son équivalent (dans ce cas, l'exhibition est médiatisée par une autre fonction textuelle). Entre autres exemples de ce dernier cas, il y a le plan extraordinaire de Gary Cooper couché dans la cabane vers la fin de L'Homme de l'Ouest, son corps remplissant momentanément l'écran en Cinémascope. Ou certaines images de Lee Marvin dans Le Point de non-retour, son corps appuyé sur une balustrade ou encadré par une porte. On peut trouver des exemples du premier cas dans le générique de fin de L'Homme de l'Ouest à nouveau (un exemple auquel Willemen se réfère) et de Junior Bonner.

La présentation de Rock Hudson dans les mélodrames de Sirk est un cas particulièrement intéressant. Il y a systématiquement des moments dans ces films où Hudson est présenté assez explicitement comme l'objet d'un regard érotique. Ce regard est en général féminin. Mais le corps de Hudson est féminisé dans ces moments-là, ce qui témoigne de la force des conventions qui imposent que seules les femmes puissent fonctionner comme l'objet d'un regard explicitement érotique. De tels cas de "féminisation" sont repérables aussi dans la comédie musicale, le seul genre du cinéma dominant dans lequel le corps masculin a pu être exhibé de façon décomplexée et cohérente (un exemple particulièrement clair et intéressant est la présentation de John Travolta dans La Fièvre du samedi soir).

29 C'est un refus de reconnaître ou de rendre explicite l'érotisme qui caractérise les trois fonctions et processus psychiques discutés ici en relation avec des images d'hommes: l'identification, le regard voyeuriste et le regard fétichiste. C'est cela qui tend pardessus tout à différencier la représentation filmique des images d'hommes et de femmes. Bien que j'ai cherché à ouvrir un espace à l'intérieur des arguments et des thèses de Laura Mulvey, pour montrer que les éléments qu'elle considère en lien avec les images de femmes peuvent et doivent aussi l'être en lien avec les images d'hommes, je suis tout à fait d'accord avec son postulat de départ qui est que le regard du spectateur de cinéma dominant est implicitement masculin: c'est l'une des raisons fondamentales pour lesquelles les éléments érotiques que comportent les relations entre le spectateur et l'image de l'homme doivent être constamment déniés et désavoués. Si ce n'était pas le cas, le cinéma dominant devrait ouvertement accepter l'homosexualité masculine qu'il cherche à dénigrer ou à nier si systématiquement. Telle quelle, l'homosexualité masculine est constamment présente de façon sous-jacente, comme un aspect potentiellement troublant de nombreux films et de genres, mais qui est traité d'une façon oblique, symptomatique, et qui doit être réprimé. Alors que le cinéma dominant, dans sa présupposition d'une norme, d'une perspective et d'un regard masculins, peut constamment prendre les femmes et l'image de la femme comme objet d'investigation, il a rarement exploré les hommes et l'image de l'homme de la même façon: les femmes sont un problème, une source d'anxiété, d'enquête obsessionnelle, les hommes ne le sont pas. Lorsque les femmes sont explorées, les hommes sont testés. La masculinité, en tant qu'idéal, au moins, est implicitement connue. La féminité, au contraire, est un mystère. C'est l'une des raisons pour lesquelles la représentation de la masculinité, à la fois au cinéma et en dehors, a été si rarement discutée. Espérons que cet article contribuera à une telle discussion.

Je voudrais remercier John Ellis et Andrew Higson pour leurs commentaires sur une version antérieure de cet article, qui est basé sur une communication donnée au 
colloque de SEPT Day sur la masculinité qui s'est tenu à Londres, au Four Corners Film Workshop, le 19 mars 1983.

\section{BIBLIOGRAPHIE}

BELlour Raymond (1975), « Le Blocage symbolique », Communications, n² 23, Paris, 1975.

CooK Pam (1982), « Masculinity in Crisis ? », Screen, vol. 23, n³ 3-4, sept-oct. 1982, p. 39-46.

CAUGHIE John \& Gillian SKIRROW (1982), « Ahab Ishmael... and Mo », Screen vol. 23, n 3-4, sept-oct. 1982, p. 54-59.

ELLIS John (1982), Visible Fictions, Londres, Routledge et Paul Kegan, 1982.

MoDLESKI Tania (1982), « Film Theory’s Detour », Screen, vol. 23, n 5, nov-déc. 1982, p. 72-79.

Mulvey Laura (1975), « Visual Pleasure and Narrative Cinema », Screen, automne 1975, vol. 16, $\mathrm{n}^{\circ} 3$, p. 6-18. Traduction intégrale en ligne http://www.debordements.fr/Plaisir-visuel-etcinema-narratif

Mulvey Laura (1981), « Afterthoughts... inspired by Duel in the Sun », Framework 15/16/17, été 1981.

NEALE Steve (1982), « Chariots of Fire, Images of Men », Screen, vol. 23, n 3-4, sept-oct. 1982, p. 47-53.

PROPP Vladimir (1970), Morphologie du conte, Paris, éditions du Seuil.

RoDowick David N. (1982), « The Difficulty of Difference », Wide Angle, vol. 5, n 1, p. 4-15.

SAFOUAN Moustapha (1981), « Is the Oedipus Complex Universal ? » m/f, $n^{\circ} 5 / 6,1981$, p. 85-87.

Willemen Paul (1981), « Anthony Mann : Looking at the Male », Framework, n 15/16/17, été 1981.

\section{NOTES}

1. Voir la traduction de Gabrielle Hardy sur le site Débordements, corrigée par mes soins. http:// www.luxonline.org.uk/articles/visual_pleasure_and_narrative_cinema(printversion).html 


\section{AUTEURS}

\section{STEVE NEALE}

Steve Neale is Research Professor in Film, Media and Communication Studies at Sheffield Hallam University. He is the author of Genre (BFI, 1980), Cinema and Technology (MacMillan, 1985) and Genre and Hollywood (Routledge, 2000), co-author of Popular Film and Television Comedy (Routledge, 1990) and co-editor of Contemporary Hollywood Cinema (Routledge, 1998) and Un-american Hollywood: Politics and Film in the Blacklist Era (Rutgers University Press, 2008) and editor of The Classical Hollywood Reader (Routledge, 2012) and Silent Features: Essays on Silent Feature-Length Films 1914-1934 (Exeter University Press, 2016). 